Diabetologe 2015 · 11:193-193

DOI 10.1007/s11428-014-1302-9

Online publiziert: 29. April 2015

(c) Springer-Verlag Berlin Heidelberg 2015

\author{
A. Zeyfang ${ }^{1,2} \cdot$ C. Sieber ${ }^{3,4}$ \\ ${ }^{1}$ AGAPLESION Bethesda Krankenhaus Stuttgart, Stuttgart \\ ${ }^{2}$ Institut für Epidemiologie, Universität Ulm, Ulm \\ ${ }^{3}$ Institut für Biomedizin des Alterns, Friedrich-Alexander-Universität Erlangen-Nürnberg, Nürnberg \\ ${ }^{4}$ Klinik für Allgemeine Innere Medizin und Geriatrie , Krankenhaus \\ Barmherzige Brüder Regensburg, Regensburg
}

\title{
Der geriatrische Patient mit Diabetes mellitus
}

\section{Besonderheiten bei der Ernährung und bei Fußproblemen}

Während sich die große Mehrheit der Publikationen zum metabolischen Syndrom und Typ-2-Diabetes insbesondere mit dem Problem der Adipositas auseinandersetzt, besteht bezüglich der letzten Phase des Lebens von Menschen mit Typ-2-Diabetes hinsichtlich Gewichtsentwicklung und Ernährungsempfehlungen noch Forschungsbedarf. Fehl- und Mangelernährung, Sarkopenie und Gebrechlichkeit sind häufige Altersprobleme bei Menschen mit Diabetes, die bewirken, dass sich die Ernährungsempfehlungen für ältere, geriatrische Menschen mit Diabetes von denen jüngerer sehr deutlich unterscheiden.

Das Autorenteam Varlemann, Feucht und Frank besteht aus Diplom-Ökotrophologinnen, Diabetesberaterinnen und Ernährungswissenschaftlern und stellt im Schwerpunktthema „Diabetes mellitus und Ernährung im Alter" besondere Vorgehensweisen zur Diagnostik des Ernährungszustandes bei älteren Patienten vor. Auch die Interaktionen mit den sog. geriatrischen Syndromen sowie konkrete Handlungsempfehlungen mit praktischen Tipps zur Ernährungsintervention werden erläutert.

Die Autoren Wernecke, Gabel und Lobmann sind Experten für die konservative, aber auch die orthopädisch-chirurgische Behandlung des diabetischen Fußsyndroms bei Älteren. Die Reduktion der hohen Anzahl von Majoramputationen um die Hälfte, insbesondere beim älteren Men- schen mit Typ-2-Diabetes, war eines der wichtigsten Ziele der St. Vinzenz-Deklaration von 1989. Auch Dank des zunehmenden Wissens um die klinisch relevanten Besonderheiten - gerade beim betagten, geriatrischen Patienten - gewinnen wir in $\mathrm{Zu}$ kunft eine Chance, durch mehr Verständnis dieser Zusammenhänge auch weiter dem Ziel näher zu kommen, Majoramputationen zu vermeiden. Die Autoren weisen auf den Einfluss der geriatrischen Funktionsstörungen, aber auch jener begleitenden Komorbiditäten hin, die bei der Behandlung des diabetischen Fußsyndroms (DFS) im Alter eine deutlich größere Rolle spielen als beim jungen Menschen. Die Unterscheidung in verschiedene Funktionskategorien erlaubt differenzierte Vorgehensweisen und Zielsetzungen - besonders beim geriatrischen Patienten mit DFS.

\section{》) Geriatrische Patienten mit Diabetes mellitus sind eine stetig wachsende Patientengruppe}

Erfreulicherweise wird für die stetig wachsende Patientengruppe der geriatrischen Patienten mit Diabetes mellitus auch zunehmend in den Fachgesellschaften Raum geschaffen, um die Probleme und Besonderheiten, aber auch die Bedürfnisse und Erwartungen dieser Menschen adäquat zu beschreiben und somit behandeln zu können. Wir hoffen auf weitere Beiträge zu diesem spannenden Thema.

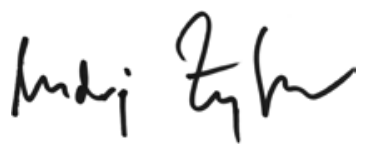

Dr. Dr. Andrej Zeyfang

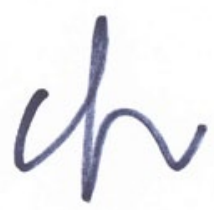

Prof. Dr. Cornel Sieber

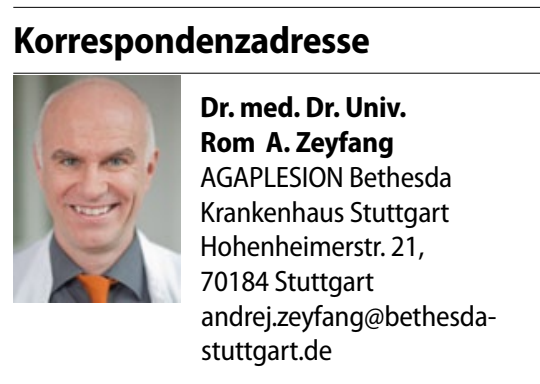

Einhaltung ethischer Richtlinien

Interessenkonflikt. A. Zeyfang erhielt Forschungsunterstützung von der Berlin-Chemie AG sowie Vortragshonorare von Berlin-Chemie, Lilly, Novo-Nordisk, Pfrimmer, Roche und Sanofi-Aventis. C. Sieber hat keinen Interessenkonflikt.

Dieser Beitrag beinhaltet keine Studien an Menschen oder Tieren. 